\title{
Nitinol Carbofilm coated stents for peripheral applications: Study in the porcine model
}

\author{
M. PRUNOTTO ${ }^{1, *}$, C. ISAIA ${ }^{2}$, M. A. GATTI ${ }^{3}$, E. MONARI ${ }^{3}$, E. PASQUINO ${ }^{2}$, \\ M. GALLONI ${ }^{4}$ \\ ${ }^{1}$ Department of Pathology \& Immunology, CMU, University of Geneva, Switzerland \\ E-mail: marco.prunotto@medecine.unige.ch \\ ${ }^{2}$ Clinical Research Department, Sorin Biomedica Cardio S.r.I., Italy \\ ${ }^{3}$ Biomaterials Laboratory, University of Modena and Reggio Emilia, Italy \\ ${ }^{4}$ Veterinary Morphophysiology Department, Biomaterials Laboratory, \\ University of Turin, Italy
}

Purpose: Testing the safety and foreign body reaction (FBR) of a nitinol self-expandable carbon-coated stent system in the porcine animal model at different follow-up (FU) periods. Methods: Fifteen minipigs received 30 carbon-coated self-expandable nitinol stents in iliac arteries. Explants were carried out at 7 ( 3 animals), 30 (4 animals), 90 (4 animals) and 180 (4 animals) day FU, for evaluation of acute, sub-acute and chronic biological response to the implanted devices. Histological, immunohistochemical, histomorphometric and scanning electron microscopy (SEM) analyses were performed to assess inflammatory reaction, endothelialization process, neointimal growth and cellular composition. Results: Thirty stents were successfully implanted. No mural thrombi were observed at gross examination or by angiography. Histologically no significant inflammatory reaction was detected: the stents appeared covered by a thin monolayer of endothelial cells even at 7 day FU. The neointima presented homogeneous growth and moderate thickness after 30,90 and 180 days explants $(0.38 \pm 0.36 \mathrm{~mm}, 0.33 \pm 0.30 \mathrm{~mm}, 0.27 \pm 0.25 \mathrm{~mm}$ respectively). Internal and external elastic laminae were intact in $95 \%$ of stented arteries. Histological data validations of vessel endothelialization was obtained with SEM for the seven day follow-up group. Conclusions: This study showed good remarkable technical performances, minimal FBR and biocompatibility comparable with other available pre-clinical experimentations.

(c) 2005 Springer Science + Business Media, Inc.

\section{Introduction}

Arterial occlusive pathologies represents the third major cause of death in developed countries. To treat these vessel stenosis, in the 80's new mini-invasive procedures were introduced. This complex of techniques, defined as interventional cardiology (IC), have dramatically modified revascularisation strategies [1]. In IC procedures arterial occlusions are treated without any surgical operation with consequent lower risk for patients and no need for long confinement in bed. At the beginning, these procedures were mainly represented by percutaneous transluminal angioplasty (PTA). In this technique a catheter containing an inflatable balloon at the distal part is advanced along the vessel and when target site is reached the lumen of the artery is widened by mean of balloon inflation. This has been proven to be effective in the acute situation; unfortunately the mechanical stress itself, caused by the balloon dilation, became a further cause of re-stenosis. In the last few years PTA has been almost completely substituted by stenting. In this case, the distal part of the catheter contains a stent, i.e. a slotted tube, that is left in place upon the inflation of the balloon. Several clinical and pre-clinical studies $[2,3]$ have demonstrated a remarkable increase in procedural success and reduced postprocedural complications of stenting vs. PTA. Despite these relevant clinical results, in-stent restenosis (ISR) is still high, ranging from $10 \%$ to $50 \%$ of all coronary stents implantations [4-7].

In-animal experimentations demonstrated that this ISR is due both to stent composition and device surface treatment. Deployment technique itself can also disrupt the endothelial cell monolayer causing platelets activation, secretion of inflammation mediators, macrophages recruitment, smooth muscle cells (SMC) activation and proliferation and extracellular matrix (ECM) deposition conducing to neointimal proliferation [8, 9].

Balloon expandable (BE) stents first developed for coronary arteries were then applied to the peripheral 
district $[10,11]$. But since the pioneer work of Dotter et al. [12] on nickel titanium alloy (nitinol) as a source for endovascular devices, it seemed clear that these metal alloys could be exploited for peripheral stent design.

As a matter of fact, Nitinol, due to its super elasticity offers a better resistance to radial forces than BE stents. Nitinol has also shape memory allowing for a specific implantation technique: the stent is kept in a collapsed condition inside a delivery catheter. After the implant site is reached, a catheter sleeve is removed so that the stent can automatically expand, reaching the vessel wall.

The aim of this in-animal study is the assessment of procedural performances and biological features in terms of restenosis evaluation of a new selfexpandable carbon coated stent (Flype Carbostent, Sorin Biomedica-Italy) in the porcine animal model.

\section{Materials and methods}

The implant procedures were performed at the Institute Mutualiste Montsouris (IMM) in Paris, in accordance with the guidelines of the National Institute of Health for the Care and Use of Laboratory Animals [13]. The histological and histomorphometric analyses were conducted at the Biomaterials Laboratory of Veterinary Medicine School at University of Turin, Italy.

\subsection{Stent description}

Flype Carbostent peripheral stents (Fig. 1) are self expandable tubular laser cut, nitinol devices with multi-segmented design with a fifteen circumferential V-shaped struts geometry providing stent expansion during deployment and radial resistance after implant; V-tracts are interconnected by longitudinal links in or- der to improve the flexibility along the stent major axis and aid an homogeneous deployment. Struts thickness is $200 \mu \mathrm{m}$ and struts width is $100 \mu \mathrm{m}$. The interconnected cellular design allows minimal foreshortening and provides an optimal lesion coverage, approximately $21 \%$ and outward radial force to the luminal vessel surface.

Flype stent was designed exploiting nitinol pseudoelasticity effect. In particular, stent geometry and Austenite finish temperature have been choosen in order to minimize the stent continuing opening force acting on the vessel wall (COF) and to maintain the vessel patency thanks to an optimal value of stent resistive force (RRF).

The over-the-wire release system (Fig. 2) consists of an external retractable sheath $(5.7 \mathrm{Fr}$ proximally, $6 \mathrm{Fr}$ distally), that contains in the distal end the stent coiled along the longitudinal axis. The proximal part of the delivery system contains an internal core for the insertion of the guide wire and a delivery system handle.

To treat the lesion the catheter is advanced to the target site. Upon retraction of the sheath the stent expands due to its superelastic properties and reach the vessel wall.

The stent is prepared with a nickel titanium alloy according to the ASTM F2063 standard. it's than laser cut, deburred, heat set and electropolished. Heat passivation is achieved at about $500{ }^{\circ} \mathrm{C}$ and the stent is shaped and fixed at its nominal diameter. The heat treatment confers the correct mechanical characteristics and induces the desired $\mathrm{TiO}_{2}$ layer at device surface; electropolishing confers the final surface finishing, the so called mirror polishing. The stent is then Carbofilm ${ }^{\mathrm{TM}}$ coated. This turbostratic carbon film has a thickness of $0.3-0.5 \mu \mathrm{m}$ and has the same structure of the pyrolitic carbon but is deposited at room temperature in high vacuum $[14,15]$. Metal implantable devices when

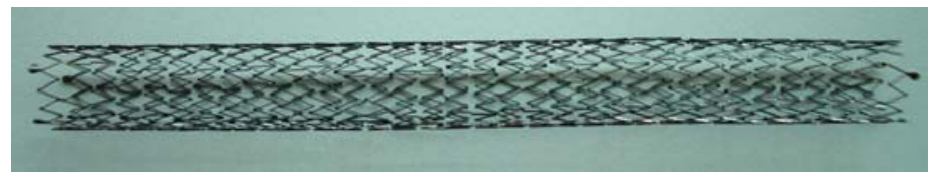

Figure 1 The Flype stent. Detail of the unconstrained stent showing strut design and tantalum radio-opaque markers.

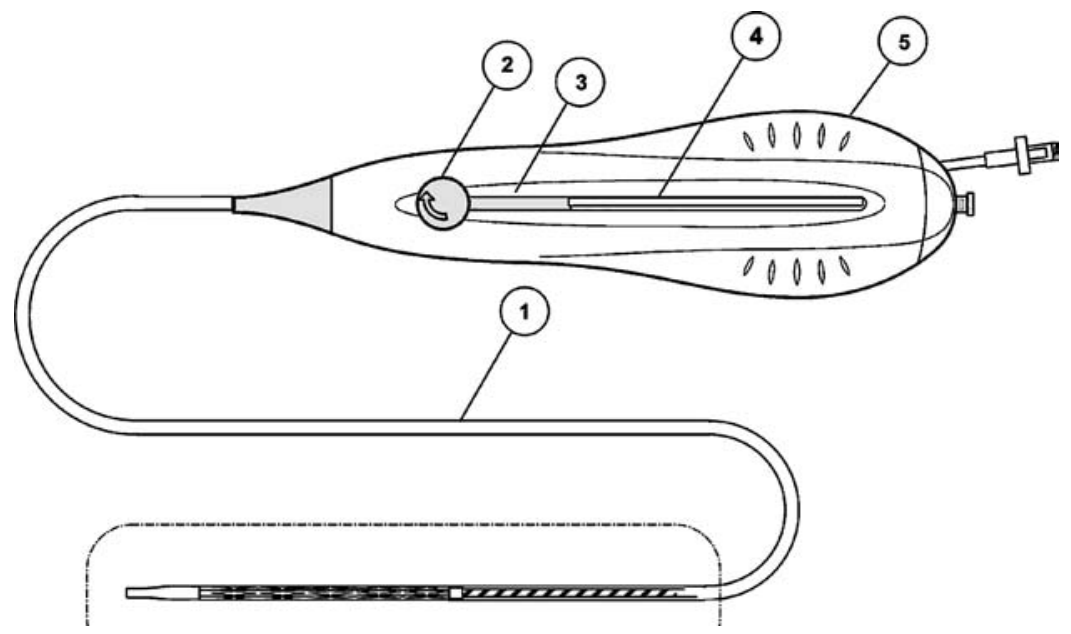

Figure 2 The stent delivery system: 1) Proximal body of the external sheath 2) Control knob 3) Built-in mechanism 4) Luer connector 5) Release system handle. 
coated with Carbofilm become similar to pyrolytic carbon in terms of tissue and blood compatibility. Three tantalum radio-opaque markers are located at each stent ends.

\subsection{Animal model}

The experimental study was performed on 15 nonatherosclerotic minipigs weighing between 35 and $45 \mathrm{Kg}$. The pigs were sacrificed at $7(n=3), 30(n=4)$, $90(n=4)$ and at 180 days $(n=4)$. All selected animals were visited three days before implants, to verify the clinical conditions. Two days before interventions they were submitted to a pharmacological treatment based on acetylsalicylic acid (ASA) (300 mg/day) and nifedipine (30 mg/day). A 24-hour food and liquids fasting before intervention was adopted.

\subsection{Stent deployment}

Animals were anesthetized by an intramuscular injection of ketamine $(10 \mathrm{mg} / \mathrm{kg})$ and azaperone $(0.5 \mathrm{mg} / \mathrm{kg})$. Deep anesthesia was induced by intravenous injection of thiopental $(10 \mathrm{mg} / \mathrm{kg})$ and maintained under a gaseous mixture of isoflurane (Abbott Laboratories, Illinois-USA) (2\%) in pure oxygen.

Iliac arteries were reached via surgically isolated right carotid artery. The procedural anticoagulation was obtained by a bolus of of heparin (100 I.U./kg) injected intravenously.

An 8F introducer (Medex Medical, Rossendale Lancashire-UK) was positioned and a $8 \mathrm{~F}$ guiding catheter Judkins right 3.5-4 (Medi-Dyne, Queensbury-NY) was introduced through this channel. An angiogram of the target vessels, by means of fluoroscopy, was obtained, using the guiding catheter as dimensional reference, in order to determine the diameter of the vessel lumen. For each frozen frame were injected $5 \mathrm{cc}$ of contrast medium. A guide-wire 0.035 " "support" (Kimal, Uxbridge-UK) was used to progress the stent delivery system to the target vessel. The stent is then automatically deployed by means of the release system handle. Stents were placed in iliac arteries without any injury prior to stent placement. Post-procedural angiogram was performed to assess the complete and correct expansion to the final nominal diameter.

All stents were implanted by the same operator. $6.0 \mathrm{~mm}$ diameter and $40 \mathrm{~mm}$ nominal length stents were tested. No post-deployment balloon dilation was applied.

Pigs were then submitted to anti-platelet treatment with ASA $300 \mathrm{mg} /$ day until the explant day. All animals survived until the planned explant day. At the end of the scheduled follow-up, each animal underwent a further angiographic control in order to assess the vessel patency. Before euthanasia, 14.000 I.U. of heparin were intravenously administrated. Animals were euthanized by an intravenous injection of barbiturate $(60 \mathrm{mg} / \mathrm{kg})$, following the guidelines of the "2000 Report of the AVMA Panel on Euthanasia" [16].

\subsection{Microscopic analysis, morphological evaluation and morphometrical analysis}

After the explant, iliac arteries were excised, washed by mean of $500 \mathrm{ml}$ of heparined physiological solution and then placed in $10 \%$ buffered formalin for $48 \mathrm{~h}$ and then washed overnight in water. Arteries underwent X-ray assessment, in order to evaluate the absence of distortion and structural failure of the stent struts. The stented portion of each artery was dehydrated in ascending concentrations of ethanol, subsequently infiltrated and embedded in methylmethacrylate plastic. The sealed vials containing stented samples were placed in $-20^{\circ} \mathrm{C}$ freezer for polymerisation.

Cross-sections, $1.5 \mathrm{~mm}$ thick, were cut from the proximal, middle and distal portion of the embedded stented specimens, using a rotating precision saw (Tronconneuse Presi P100, Grenoble, France). Struts were manually removed from these cross-sections in order to allow the preparation for microtome cutting. The sections were then glued on a specimen holder; $5 \mu \mathrm{m}$ slices were cut with a tungsten carbide blade microtome (Microtome 2135, Leica, Heerbrugg) and stained using Mayer's emallume/eosine-floxine and Movat pentachrome stain. The vessel segments proximal and distal to the stents were embedded in paraffin, sectioned and stained as described above.

Neointimal thickness was measured as the distance from internal elastic lamina (IEL) to the luminal border both at and between each stent strut.

The injury score [17] was calculated for each stent strut; the presence of granulomas, vacuoles and neoangiogenesis were also identified for each stent strut.

The neointimal inflammation score [18], the presence and rate of inflammatory cells in the neointimal layer, was evaluated in five randomly chosen $40 \times$ microscopic fields adjacent to the stent struts and neointimal inflammatory response was graded as: 0 (no inflammation), 1 (0-500 inflammatory cells $\left./ \mathrm{mm}^{2}\right), 2$ (500-800 inflammatory cells $/ \mathrm{mm}^{2}$ ) and 3 (over 800 inflammatory cells $/ \mathrm{mm}^{2}$ ).

Immunohistochemistry (IHC) was performed on paraffin sections obtained after struts removal for all collected samples. Macrophages (CD68, Dako, Carpinteria, CA, USA) and von Willebrand factor (Dako) were targeted in 7-days FU group. Alpha-actin (Dako) for smooth muscle cells (SMC) identification was performed for each group of FU for a qualitative evaluation of neointima and media composition.

Scanning electron microscopy (SEM) was performed on 7-day FU group. Specimens were dehydrated, longitudinally cut, critical point dried (BAL-TEC AG, Föhrenweg, Liechtenstein), and finally gold coated (BAL-TEC AG). Specimens were observed with a Cambridge S200 (LEO, Cambridge, UK) at operating voltage varying from 15 to $20 \mathrm{kV}$ and $1536 \times 1200$ pixels; digital images were acquired via Pixie-3000, a real time active SEM framestore (Deben software, Stowupland, UK).

Histomorphometric analysis was performed using an image analysis software (Image Pro Plus; Media Cybernetics, Silver Spring, MD) on stented segments and 
on correspondent proximal and distal portion of iliac arteries. For each stented section the following parameters were calculated: lumen area, area within internal elastic lamina (IEL) and area within external elastic lamina (EEL). Medial area was obtained subtracting the EEL area from IEL area, while neointimal area was acquired as a subtraction of the lumen area from the IEL area. The percentage of stenosis was calculated following the formula [10]:

$$
\% \text { AREA STENOSIS }=\frac{[\mathrm{IEL}-\mathrm{L}]}{\mathrm{IEL}} \times 100
$$

All continuous histomorphometric variables were expressed as mean \pm standard deviation.

\section{Results}

3.1. Macroscopic and angiographic findings 30 stents were successfully implanted in orthotopic position. The angiographic controls (Fig. 3(a)) performed after stent deployment showed stents expanded to the nominal final diameter, pre-explant angiogram (Fig. 3(b)) showed no acute or subacute stent thrombosis at short and mid term follow-ups ( 7 and 30 days). A moderated neointimal proliferation was observed in 90 and 180 day FU groups. At X-ray assessment no stent distortions or structural fractures were found.

\subsection{Light microscopy and morphometry}

Microscopic evaluation was performed on all stented vessels, subdivided in 4 different periods of follow-up. A total of twenty seven arteries specimens were submitted to histological examination (3 arteries from 7-day FU group, 8 arteries from the 30-day FU group, 8 arteries from the 90-day FU group and 8 arteries from the 180-day FU group). For a complete set of morphometrical data regarding Flype stents see Table I.

\subsubsection{7-day follow-up}

At 7 day FU 3 animals were sacrificed and three stents were submitted to SEM analysis and three to histological evaluation. Vessels were fully endothelialized (Figs. 4(a), 5(a), and 6), moderate symmetrical neointimal proliferation (neointimal thickness of $0.04 \pm$
TABLE I Flype stents-morphometric analysis

\begin{tabular}{lcccc}
\hline & 7 days FU & 30 days FU & 90 days FU & 180 days FU \\
\hline IEL $\left(\mathrm{mm}^{2}\right)$ & $19.26 \pm 1.77$ & $17.70 \pm 2.03$ & $18.19 \pm 2.55$ & $16.64 \pm 1.40$ \\
EEL $\left(\mathrm{mm}^{2}\right)$ & $22.60 \pm 1.76$ & $20.53 \pm 2.32$ & $21.13 \pm 3.12$ & $19.13 \pm 1.65$ \\
Lumen area & $17.79 \pm 1.81$ & $13.10 \pm 3.35$ & $13.23 \pm 3.26$ & $12.50 \pm 2.92$ \\
$\left(\mathrm{~mm}^{2}\right)$ & & & & \\
$\%$ stenosis & $7.67 \pm 1.70$ & $26.41 \pm 15.12$ & $27.16 \pm 14.50$ & $25.08 \pm 15.09$ \\
$\begin{array}{l}\text { Neointimal } \\
\text { thickness } \\
(\text { mm) }\end{array}$ & $0.04 \pm 0.02$ & $0.27 \pm 0.21$ & $0.33 \pm 0.30$ & $0.27 \pm 0.25$ \\
$\begin{array}{l}\text { Neointimal } \\
\text { area (mm }\end{array}$ & $1.47 \pm 0.29$ & $4.60 \pm 2.70$ & $4.86 \pm 2.81$ & $4.14 \pm 2.56$ \\
$\begin{array}{l}\text { Injury Score } \\
\text { 0 }\end{array}$ & & $0.09 \pm 0.22$ & $0.10 \pm 0.18$ & $0.31 \pm 0.48$ \\
\hline
\end{tabular}

Legend $:$ IEL $=$ Internal Elastic Lamina; EEL = External Elastic Lamina.

$0.02 \mathrm{~mm}$, neointimal area of $\left.1.47 \pm 0.29 \mathrm{~mm}^{2}\right)$, no particular inflammatory response (inflammation score $0.00 \pm 0.00)$ was detected, small blood clots and vacuoles were detected only around stent struts. Internal elastic lamina (IEL) and external elastic lamina (EEL) were always intact (injury score $0.00 \pm 0.00$ ).

\subsubsection{0 day follow-up}

At 30 day FU 4 animals were sacrificed; (Figs. 4(b) and 5(b)), histological findings showed complete vessel endothelialization. A symmetrical neointimal proliferation was observed in four specimens; the neointimal growth was asymmetrical in the remaining ones. Just in 1 stent a remarkable neointimal proliferation was detected (neointimal thickness $1.15 \pm 0.21 \mathrm{~mm}$ and neointimal area $14.12 \pm 2.68 \mathrm{~mm}^{2}$, injury score $0.03 \pm 0.03$ ) together with vacuoles and blood clots around the stent struts, whereas the remaining stents showed mean neointimal thickness of $0.27 \pm 0.21 \mathrm{~mm}$, neointimal area $4.60 \pm 2.70 \mathrm{~mm}^{2}$ and injury score $0.09 \pm 0.22$. Neointima was mainly composed by SMC embedded in proteoglican matrix. No inflammatory reaction was detected (inflammation score $0.00 \pm 0.00$ ).

IEL and EEL were always intact and media appeared regular in all the examined specimens.

\subsubsection{0 day follow-up}

At 90 day FU 4 animals were sacrificed; all stents were subjected to histological study (Figs. 4(c) and $5(\mathrm{c}))$. The arteries showed mean neointimal
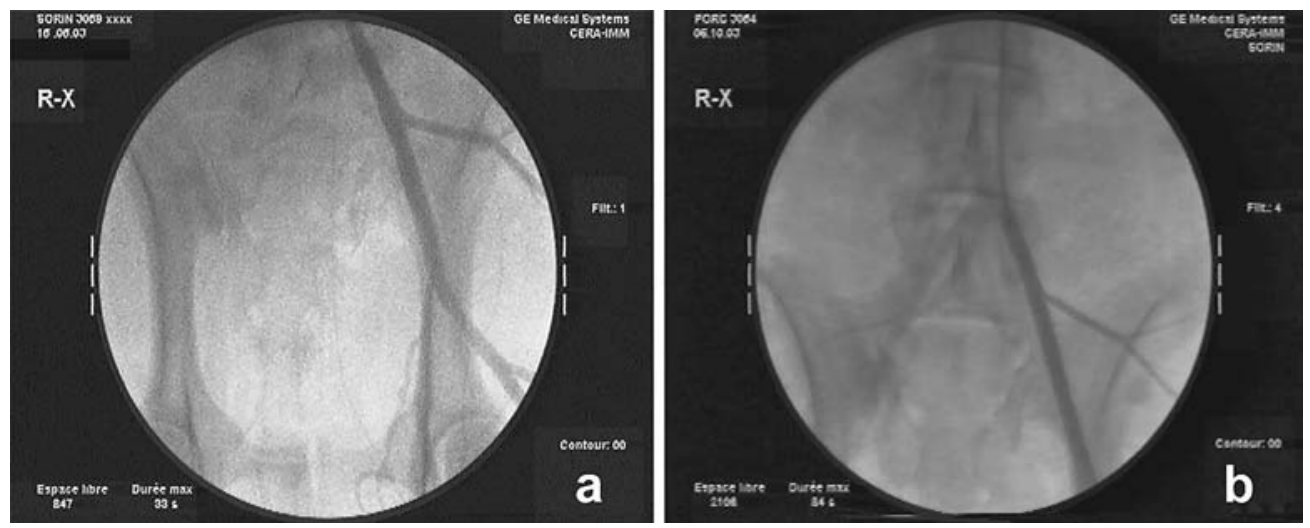

Figure 3 a) Post procedural angiogram of implanted iliac artery - b) Long term follow up pre-explant angiorgram (180 day FU) showing the vessel patency. 

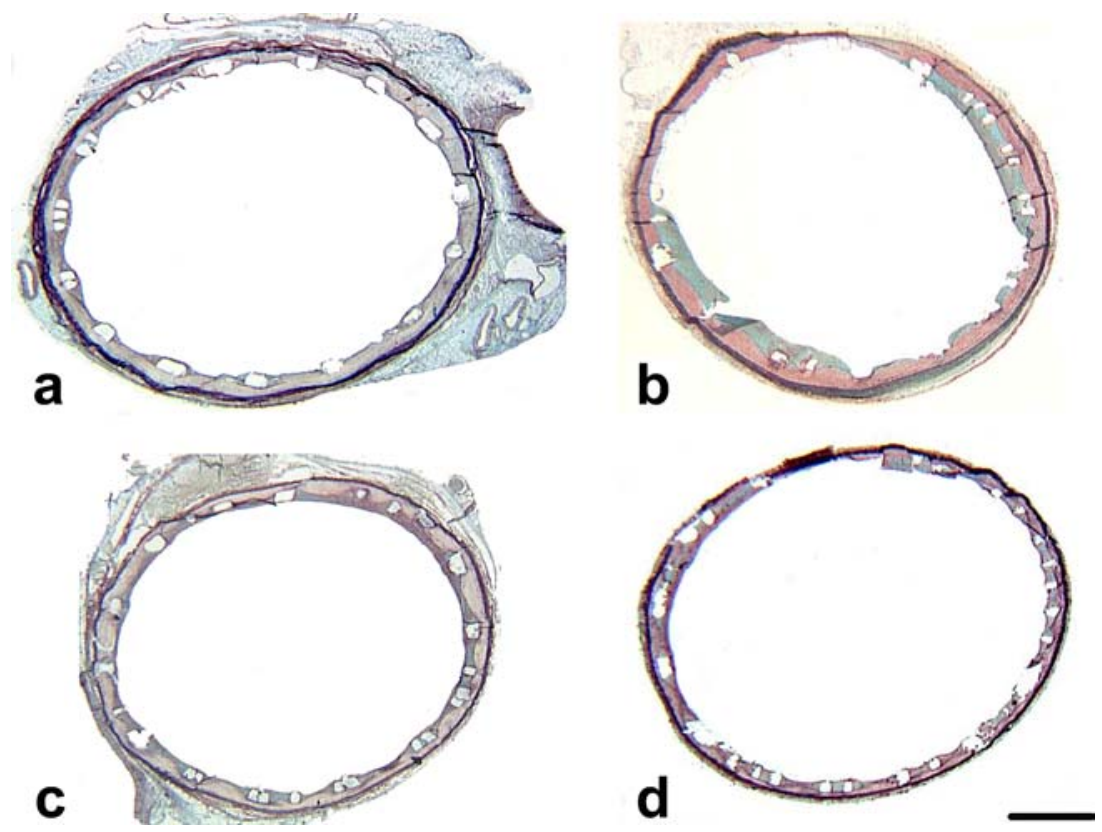

Figure 4 Flype stent-full vessel images (magnification circa 20×), Histological sections at a) 7 days FU b) 30 days FU c) 90 days FU and d) 180 days FU, Movat pentachrome stain, bar lenght $=1 \mathrm{~mm}$.
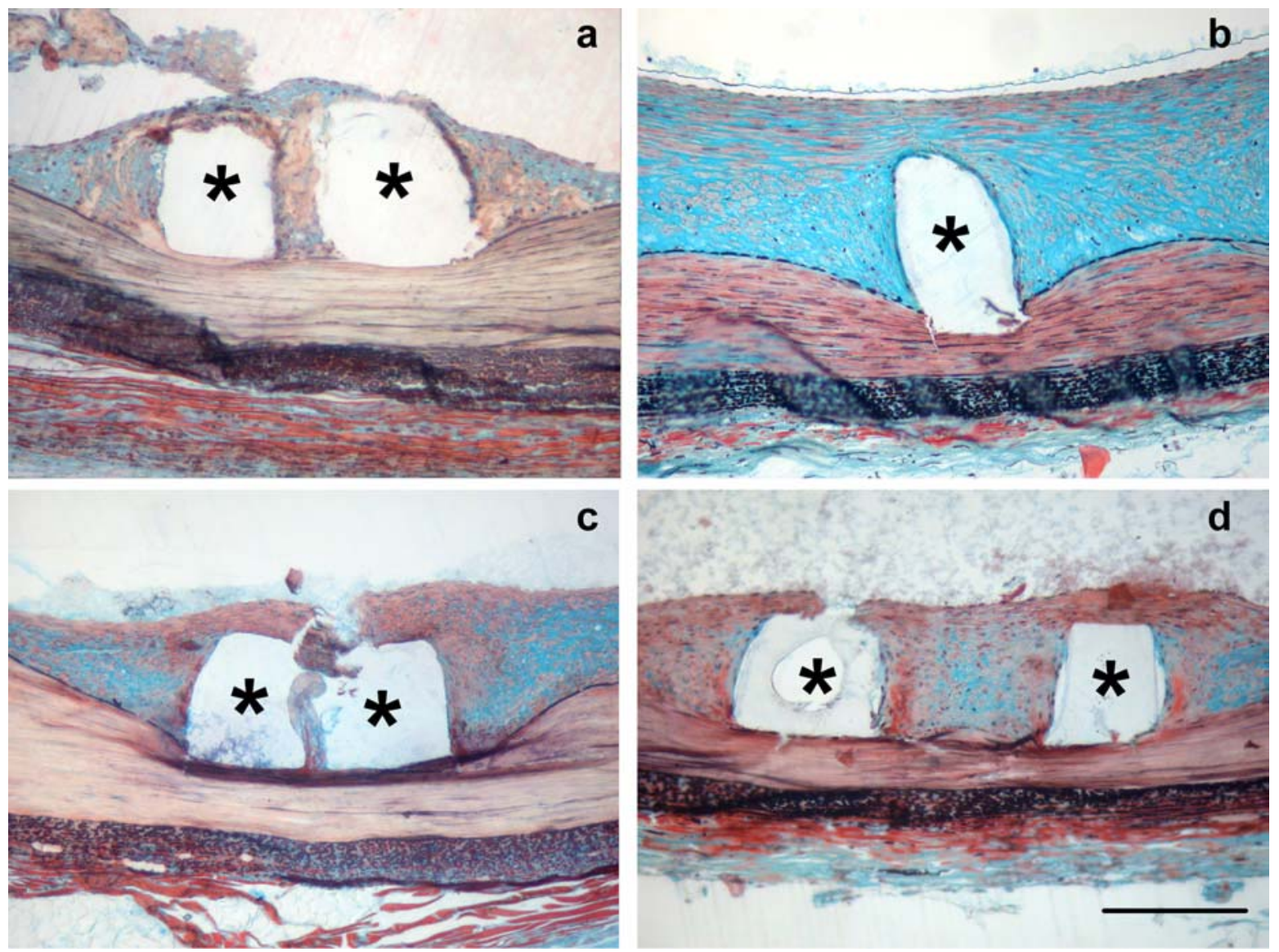

Figure 5 Flype stent-Detail of stent struts, $150 \times$ a) 7 days FU b) 30 days FU c) 90 days FU and d) 180 days FU, Movat pentachrome stain, bar lenght $=150$ micron. Asterisk indicates the stent struts.

thickness of $0.33 \pm 0.30 \mathrm{~mm}$ and neointimal area of $4.86 \pm 2.81 \mathrm{~mm}^{2}$. Generally all stents showed symmetrical neointimal proliferation but in the case of two stents, belonging to the same animal, a remarkable asymmetrical neointimal growth was observed. Neointima was mainly composed of SMC embedded in a proteoglican matrix. In three specimens the IEL was disrupted by the stent struts (injury score $0.10 \pm 0.18$ ).
There was no evidence of inflammatory process (inflammation score $0.00 \pm 0.00$ ).

\subsubsection{0 day follow-up}

At 180 day FU 4 animals were sacrificed; all eight arteries collected (Figs. 4(d) and 5(d)), four arteries showed symmetrical neointimal growth while four arteries had 


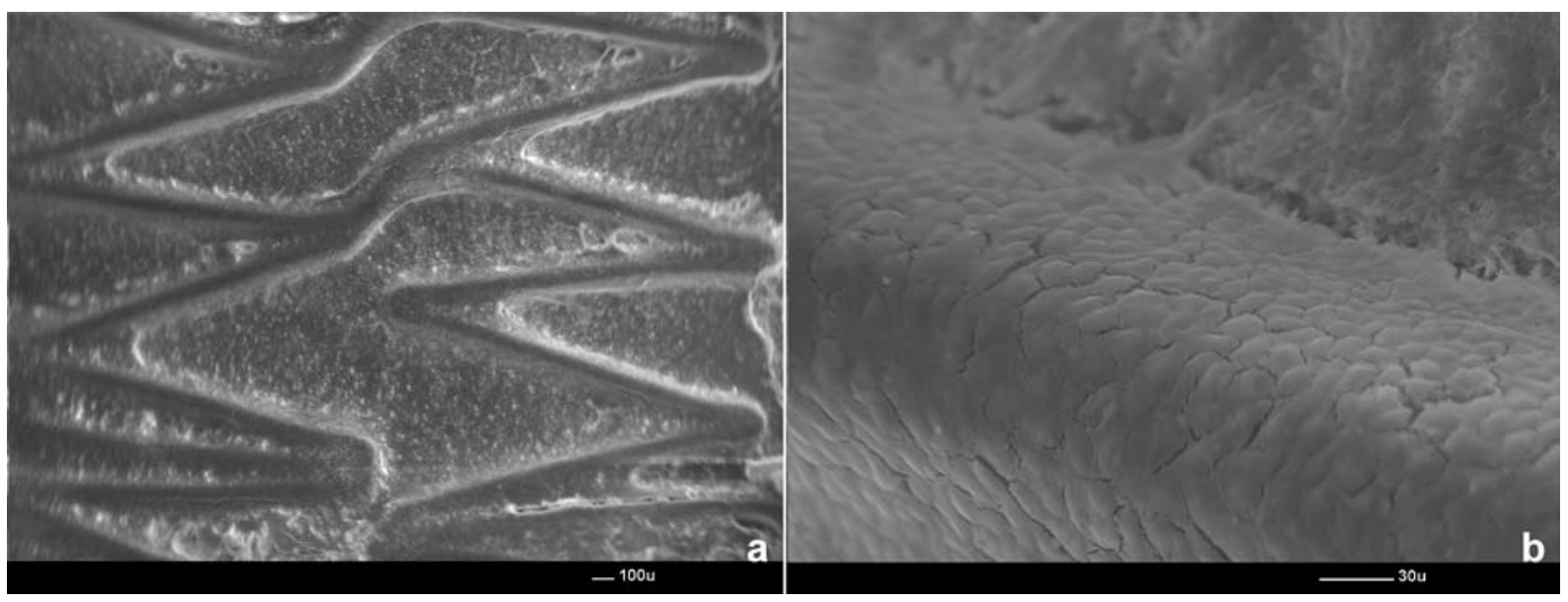

Figure 6 SEM of 7 days FU Flype stents a) Confluent and regular carpet of neo-endothelial cells, $31 \times$ b) Detail of the previous image showing neo-endothelial cells with the typical rhomboid shape covering stent strut, $340 \times$.

a slightly asymmetrical proliferation (neointimal thickness of $0.27 \pm 0.25 \mathrm{~mm}$ and neointimal area of $4.14 \pm$ $2.56 \mathrm{~mm}^{2}$ ). IEL and EEL were always intact except in one stent, where the IEL was stressed by stent struts (injury score $0.31 \pm 0.48$ ). No chronic inflammatory reaction was detect at stent struts level (inflammation score $0.28 \pm 0.461)$.

\subsection{Immunohistochemistry}

Immunohistochemistry for Von Willebrand factor on 7 days FU group revealed full endothelialization of stented arteries with a positivity of neo-endothelial cells. Rare CD68 positive cells were found in 7 days FU and this positivity was absent at 30 and 180 day FU

confirming a moderate inflammatory response in the short term. Neointima showed an intense staining for alpha actin particularly in 28 days FU group.

\subsection{Scanning electron microscopy}

The examination of three 7 day FU group iliac stents (Fig. 6) confirmed the presence on the struts of a confluent and regular carpet of neo-endothelial cells with the typical rhomboid shape; only a negligible amount of platelets and inflammatory cells were detectable.

\section{Discussion}

The introduction of the nitinol as a source for endovascular devices has provoked some concerns in the medical community due to the its high nickel content; the toxic effect of nickel is in fact well known and it's mainly related to the production of reactive oxygen species due to oxidation and redox cycling between $\mathrm{Ni}^{3+}$ and $\mathrm{Ni}^{2+}$ ions [19]. Recent studies [20] have demonstrated that heat passivation and electropolishing techniques of nitinol stents result in formation of an enriched $\mathrm{TiO}_{2}$ layer on the device surface which inhibits the redox cycling of $\mathrm{Ni}$ and consequently reduces the amount of oxidative stress directed against endothelial cells. Further pre-clinical investigations [21-23] have proven that nitinol has a biocompatibility comparable or even superior to stainless steel stents.
In the case of the present study the examined stents were heat passivated, electropolished and Carbofilm coated; the last layer being the real interface with the biological tissues. Carbofilm coated devices become similar to pyrolytic carbon in terms of tissue and blood compatibility [24]; Evidence of safety and effectiveness of this coating comes from long-term clinical experience in heart valve implants and stents implants [15].

In our study all stents were tested in a normocholesterolemic pig model with no previous injury or oversizing of the implanted devices. The pig model was adopted as in comparison with other animal models, e.g. the dog, it responds to a vessel wall injury by developing a thick neointima virtually identical to the hyperplasia found in humans in terms of cell size, cell density and histopathological appearance. Major model limitations consist of the use of disease-free arteries instead of atherosclerotic ones. A full atheroscleroticrestenosis animal model is not yet available. Although neointima formation through SMC proliferation, migration and ECM secretion is identical to humans and at the moment the pig model is the gold standard for stent pre-clinical evaluation.

All collected vessels were patent at explant, no mural thrombi were observed. All implanted stents were correctly deployed in the vessel without any malapposition and endothelialization, that has been proven to be crucial in limiting neointimal formation, was complete at 7-day FU with rhomboid-like neo-endothelial cells covering stent struts as shown from SEM and IHC results.

The tested stents presented (graph 1) a typical curve for measured morphometrical parameters with a neointimal thickness, area and \% stenosis higher at 30 day $\mathrm{FU}$, in response to the inflammation which normally has a peak circa at 7 day $[25,26]$ and decreasing values for longer FU suggesting a mechanism of vessel remodelling mainly due to the ECM as the SMC population of the neointima remains practically stable at different $\mathrm{FU}$ $\left(4341 \pm 788\right.$ cells $/ \mathrm{mm}^{2}$ at 30 day FU, $4792 \pm 1201$ cells at 90 and $4758 \pm 1278$ cells $/ \mathrm{mm}^{2}$ at 180 day FU) with no statistically significant difference.

The moderate 180 day FU neointimal thickness and cellularity results are particularly interesting as this FU 
is considered to be akin to two or three years response in humans [27]. This was clearly detectable also in the images at high magnification (Fig. 5(b)). IEL area values were not significantly different between 30 day FU and 180 day FU suggesting a good behaviour in terms expansion properties even at longer FU with no late expansion. In a recent study by Taylor et al. [28] nitinol devices in fact showed a tendency to late expansion and there is some evidence that a prolonged expansion could act as a continuous inflammatory stimulus resulting in a larger amount of neointima formation thus cancelling the nitinol advantage for no high pressure balloon deployment.

IHC results have shown no particular inflammatory response other than a normal FBR in acute FU with sparse macrophages around stents struts. Macrophage positivity decreases in longer FU confirming absence of chronic inflammation. Staining for alpha actin (a specific SMC marker) confirmed histological results of neointima composition. At 28 day FU Movat pentachrome stain revealed a marked ECM which decrease in longer FU as observed from morphometrical data analysis.

A comparison with other pre-clinical trials is particularly difficult, due to the different animal models, experimental settings, stent types, stent designs, diets, injury models and procedure manoeuvres adopted.

In that context a direct comparison is impossible; a wider comparison could be done and present study results compared, in terms of neointimal thickness and injury score at 30 and 180 day FU with a similar pre-clinical trial in the porcine iliac arteries by Verheye et al. [29]. The tested stents resulted in values of neointimal thickness and injury score respectively of $0.27 \pm 0.21 \mathrm{~mm}$ and $0.09 \pm 0.22$ for 30 day FU and $0.27 \pm 0.25 \mathrm{~mm}$ and $0.31 \pm 0.48$ for 180 day FU while for the EndoStent tested by Verheye results were respectively $0.65 \pm 0.13 \mathrm{~mm}$ and $0.86 \pm 0.05$ for 30 day FU and $0.86 \pm 0.18 \mathrm{~mm}$ and $1.21 \pm 0.17$ for 180 day FU. Results for the studied stents were similar or even better at each FU but it must be pointed out that in Verheye's work a injury was provoked just before stent implantation by balloon overstretch (balloon artery ratio 1:1.2).

Neointimal thickness results can be compared to the work of Froelich et al. [30] whose results were respectively $1.105 \pm 0.485 \mathrm{~mm}$ at 90 days FU and $1.212 \pm 0.167 \mathrm{~mm}$ at 180 day FU. Also in this case a direct comparison is incorrect, in fact an atherogenic diet was adopted for 6 weeks to increase blood cholesterol values in order to mimic the situation in humans; on day 14 of that diet an injury was provoked by means of inflation and pull back for three times of a Fogarty balloon catheter. A last comparison can be drawn with Harnek et al. [31], assuming its group 3 for reference, represented by $30 \%$ oversized self expandable stents implanted without any previous PTA, and the 56 day FU (an intermediate value between the 30 and 90 day FU of the present study) results were $2.69 \pm 1.97 \mathrm{~mm}^{2}$ for neointimal area and $0.26 \pm 0.08$ for injury score. In that case results from Harnek were comparatively better in terms of neointimal area but not in terms of injury score.

\section{Conclusions}

All findings obtained through morphological, IHC, SEM and morphometrical analysis in this pre-clinical investigation in the normocholesterolemic porcine model, i.e.: absence of mural thrombi, low inflammatory response at acute and chronic FU, early reendothelialization, reduced neointimal proliferation decreasing with longer FU suggest that these devices were safe and presented acceptable level of biocompatibility comparable with other already published in vivo cited works, bearing in mind the underlined differences in terms of oversizing and injury model adopted.

The biocompatibility of that stent together with zero risk of balloon rupture and no late expansion together with superelastic properties, suggest a future use in clinical experimentation and practice.

\section{References}

1. D. L. Fischman, M. B. LEON, et al., N. Engl. J. Med. 331(8) (1994) 496.

2. M. CEJNA, S. THURnher, et al., J. Vasc. Interv. Radiol. 12(1) (2001) 23.

3. A. Kastrati, A. SChomig, et al., Circulation 102(21) (2000) 2593.

4. M. B. LEON, P. S. TEIRSTEIN, etal., N. Engl. J. Med. 344 (2001) 250.

5. S. L. GOldBerg, A. LOUSSARARiAn, et al., J. Am. Coll. Cardiol. 37 (2001) 1019.

6. J. AL SUWAIDI, P. BERGER, et al., JAMA 284 (2000) 1828.

7. F. V. TILL, D. AliABADI, et al., Circulation 94 (1996) I332.

8. S. P. KARAS, M. B. GRA VANis, etal., J. Am. Coll. Cardiol. 20 (1992) 467.

9. A. J. CARTER, J. R. LAIRD, et al., ibid. 27 (1996) 1270.

10. P. W. SERRUYS, B. N. STRAUSS, et al., N. Engl. J. Med. 324 (1991) 13.

11. G. R. Dussaillant, G. S. Mintz, et al., J. Am. Coll. Cardiol. 26 (1995) 720

12. C. T. DOtTER, R. W. BUSCHMANN, et al., Radiology 147(1) (1983 Apr) 259

13. Institute of Laboratory Animal Resources Commission on Life Sciences, Guide for the Care and Use of Laboratory Animals (National Academy Press, Washington, 1997).

14. C. PACCAGnella, G. MAJini, et al., J. Artif. Organs 9 (1986) 115.

15. M. GAlloni, M. PRUnotto, etal., J. Vasc. Interv. Radiol. 14(8) (2003) 1053.

16. 2000 Report of the AVMA Panel on Euthanasia. JAVMA 218 (2001) 669.

17. R. S. SChWARTZ, K. C. HuBer, et al., J. Am. Coll. Cardiol. 19(2) (1992) 267.

18. G. SAngiorgi, E. ARBUSTINi, et al., Cardiovasc Intervent Radiol. 24(4) (2001) 260.

19. A. K. DUTTA, M. MISRA, et al., Carcinogenesis 13 (1992) 283.

20. S. D. PLANT, M. GRANT, et al., Biomaterials 26(26) (2005) 5359 .

21. J. RYHANEN, "Biocompatibility Evaluation of Nickel-Titanium Shape Memory Metal Alloy," (Oulu University Press, 1999).

22. B. THIERRY, M. TAB RIZIAN, et al., J. Biomed. Mater Res. 51(4) (2000) 685.

23. B. THIERRY, Y. MERHI, et al., Biomaterials 23(14) (2002) 2997.

24. A. D. HAUBOLD, H. S. Shim, et al., in edited by D.F. Williams (ed.), "Carbon in Metal Devices," (CRC Press, Boca Raton, FL, 1981).

25. A. J. CARTer, J. R. LAird, et al., J. Am. Coll. Cardiol. 24(5) (1994) 1398.

26. R. S. SCHWARTZ, N. A. CHRONOS, ibid. 44 (2004) 1373. 
27. VIRMANi, FD KOLODGIE, et al., Heart 89(2) (2003 Feb) 133.

28. A. J. TAYLOR, P. D. GORMAN, etal., Catheter Cardiovasc Interv. 53(3) (2001) 420.

29. S. VERHEYE, M. Y. SALAME, et al., ibid. 48(3) (1999) 316.

30. J.J. FROELICH, H. ALFKE, et al., J. Vasc. Interv. Radiol.
10(3) (1999) 329

31. J. HARNEK J, E. ZOUCAS, et al., Cardiovasc Intervent Radiol. 25(5) (2002) 430.

Received 30 June and accepted 19 August 2005 